\title{
Note on abstract elliptic equations with nonlocal boundary in time condition
}

Ho Thi Kim Van a

${ }^{a}$ Division of Applied Mathematics, Thu Dau Mot University, Binh Duong Province, Vietnam

\begin{abstract}
Our main purpose of this paper is to study the linear elliptic equation with nonlocal in time condition. The problem is taken in abstract Hilbert space $H$. In concrete form, the elliptic equation has been extensively investigated in many practical areas, such as geophysics, plasma physics, bioelectric field problems. Under some assumptions of the input data, we obtain the well-posed result for the solution. In the first part, we study the regularity of the solution. In the second part, we investigate the asymptotic behaviour when some paramteres tend to zero.
\end{abstract}

Keywords: Cauchy problem, elliptic equations, well-posedness, regularity.

2010 MSC: 35R11, 35B65, 26A33.

\section{Introduction}

Let $H$ be a Hilbert space. Let $\mathcal{L}: D(\mathcal{L}) \subset H \rightarrow H$ be a positive-definite, self-adjoint operator with compact inverse on $H$. Let us assume that $A$ admits an orthonormal eigenbasis $\left\{\varphi_{k}\right\}_{k \geq 1}$ in $H$, associated with the eigenvalues of the operator $\mathcal{L}$ and

$$
0<\lambda_{1} \leq \lambda_{2} \leq \cdots \lambda_{j} \leq \cdots
$$

and $\lim _{j \rightarrow \infty} \lambda_{j}=\infty$. Let $T>0$ be a given real number. In this paper, we consider the nonlinear elliptic equation

$$
\left\{\begin{array}{l}
\frac{\partial^{2} u}{\partial y^{2}}=\mathcal{L} u+F(y), y \in(0, T) \\
u_{y}(0)=0, \quad \in(0, T) \\
\alpha u(T)+\epsilon u(0)=f
\end{array}\right.
$$

Email address: hothikimvan@tdmu.edu.vn (Ho Thi Kim Van) 
where $f$ and $F$ called input data and defined later. The problem (1.1) may called abstract elliptic equations with nonlocal boundary in time condition. Non-local boundary value issues are undoubtedly one of the areas that excel in many different fields of application, such as chaos, chemistry, biology, and physics.

In problem (1.1), if $\alpha=0, \epsilon=1$ then we called the Cauchy problem for elliptic problem which also has been studied in many paper, for example [13, 14, 18, 19, 20, 21]. In the abstract framework of operators on Hilbert spaces, regularization techniques are developed by B. Kaltenbacher et al [15, 16, 17] .

To the best of our knowledge, there are not any paper concern to Problem (1.1). Our work is probably one of the first results on this type of problem for elliptic equations. Our contribution for this paper are described as follows

- The first contribution is the investigation of the solution space and the regularity of the solutions.

- The second contribution is to demonstrate the convergence of solutions when the parameters reach zero.

\section{Nonlocal in time elliptic equation}

For positive number $r \geq 0$, we also define the Hilber scale space

$$
D\left(A^{s}\right)=\left\{w \in H: \sum_{j=1}^{\infty} \lambda_{j}^{2 s}\left\langle w, \psi_{j}\right\rangle^{2}<+\infty\right\},
$$

with the following norm $\|u\|_{D\left(A^{s}\right)}=\left(\sum_{j=1}^{\infty} \lambda_{j}^{2 s}\left|\left\langle u, \psi_{j}\right\rangle\right|^{2}\right)^{\frac{1}{2}}$. Let us also define the space of Geverey type $\mathscr{V}_{s, T}$ be as follows

$$
\mathscr{V}_{s, T}=\left\{w \in H: \sum_{j=1}^{\infty} \lambda_{j}^{2 s} e^{2 \sqrt{\lambda_{j}} T}\left\langle w, \psi_{j}\right\rangle^{2}<+\infty\right\},
$$

for $s \in \mathbb{R}, T>0$. The associated norm on $\mathscr{V}_{s, T}$ is given by

$$
\|u\|_{\mathscr{V}_{s, T}}=\left(\sum_{j=1}^{\infty} \lambda_{j}^{2 s} e^{2 \sqrt{\lambda_{j}} T}\left\langle w, \psi_{j}\right\rangle^{2}\right)^{\frac{1}{2}}
$$

Theorem 2.1. Let $u^{*}$ be the solution of Problem (1.1) with the case $\alpha=1, \epsilon=0$. Let $f$ be the function belongs to $D\left(A^{\nu-\frac{\theta}{2}}\right)$ and $F \in L^{\infty}\left(0, T ; \mathscr{V}_{\nu-\theta / 2-1 / 2, T}\right)$ for any $0<\theta<1$ and $\nu>0$. Then we get $u^{*} \in$ $L^{1}\left(0, T ; D\left(A^{\nu}\right)\right)$ and the following estimate holds

$$
\begin{aligned}
\left\|u^{*}\right\|_{L^{1}\left(0, T ; D\left(A^{\nu}\right)\right)} & \leq \frac{2 C_{\theta} T^{1-\theta}}{1-\theta}\|f\|_{D\left(A^{\left.\nu-\frac{\theta}{2}\right)}\right.}+T^{2}\|F\|_{L^{\infty}\left(0, T ; \mathscr{W}_{\nu-1 / 2, T}\right)} \\
& +\frac{2 \sqrt{T} C_{\theta} T^{1-\theta}}{1-\theta}\|F\|_{L^{\infty}\left(0, T ; \mathscr{W}_{\nu-\frac{\theta-1}{2}, T}\right)} .
\end{aligned}
$$

Proof. The mild solution of Problem (1.1) in the case of $\alpha=1, \epsilon=0$ is given by

$$
\begin{aligned}
u^{*}(y) & =\sum_{j=1}^{\infty} \frac{\cosh \left(\sqrt{\lambda_{j}} y\right)}{\cosh \left(\sqrt{\lambda_{j}} T\right)}\left\langle f, \psi_{j}\right\rangle \psi_{j}+\sum_{j=1}^{\infty}\left(\int_{0}^{y} \frac{\sinh \left(\sqrt{\lambda_{j}}(y-s)\right)}{\sqrt{\lambda_{j}}} F_{j}(s) d s\right) \psi_{j} \\
& -\sum_{j=1}^{\infty} \frac{\cosh \left(\sqrt{\lambda_{j}} y\right)}{\cosh \left(\sqrt{\lambda_{j}} T\right)}\left(\int_{0}^{T} \frac{\sinh \left(\sqrt{\lambda_{j}}(T-s)\right)}{\sqrt{\lambda_{j}}} F_{j}(s) d s\right) \psi_{j} \\
& =\mathscr{I}_{1}(y)+\mathscr{I}_{2}(y)+\mathscr{I}_{3}(y) .
\end{aligned}
$$


For the term $\mathscr{I}_{1}$, using the inequality

$$
\frac{\cosh \left(\sqrt{\lambda_{j}} y\right)}{\cosh \left(\sqrt{\lambda_{j}} T\right)} \leq 2 e^{\sqrt{\lambda_{j}}(y-T)} \leq 2 C_{\theta} \lambda_{j}^{-\theta / 2}(T-y)^{-\theta}, \quad 0<\theta<1,
$$

we get the following estimate

$$
\begin{aligned}
\left\|\mathscr{I}_{1}(y)\right\|_{D\left(A^{\nu}\right)}^{2} & =\sum_{j=1}^{\infty} \lambda_{j}^{2 \nu}\left(\frac{\cosh \left(\sqrt{\lambda_{j}} y\right)}{\cosh \left(\sqrt{\lambda_{j}} T\right)}\right)^{2}\left\langle f, \psi_{j}\right\rangle^{2} \\
& \leq 4\left|C_{\theta}\right|^{2}(T-y)^{-2 \theta} \sum_{j=1}^{\infty} \lambda_{j}^{2 \nu-\theta}\left\langle f, \psi_{j}\right\rangle^{2}=4\left|C_{\theta}\right|^{2}(T-y)^{-2 \theta}\|f\|_{D\left(A^{\nu-\frac{\theta}{2}}\right.}^{2} .
\end{aligned}
$$

Hence, we obtain

$$
\left\|\mathscr{I}_{1}(y)\right\|_{D\left(A^{\nu}\right)} \leq 2 C_{\theta}(T-y)^{-\theta}\|f\|_{D\left(A^{\nu-\frac{\theta}{2}}\right)} .
$$

The second term $\mathscr{I}_{2}$ is bounded by

$$
\begin{aligned}
\left\|\mathscr{I}_{2}(y)\right\|_{D\left(A^{\nu}\right)}^{2} & =\sum_{j=1}^{\infty} \lambda_{j}^{2 \nu}\left(\int_{0}^{y} \frac{\sinh \left(\sqrt{\lambda_{j}}(y-s)\right)}{\sqrt{\lambda_{j}}} F_{j}(s) d s\right)^{2} \\
& \leq \sum_{j=1}^{\infty} \lambda_{j}^{2 \nu-1} y \int_{0}^{y}\left(\sinh \left(\sqrt{\lambda_{j}}(y-s)\right)\right)^{2}\left|F_{j}(s)\right|^{2} d s
\end{aligned}
$$

Noting that for $y \in[0, T]$, we get

$$
\left|\sinh \left(\sqrt{\lambda_{j}}(y-s)\right)\right| \leq e^{\sqrt{\lambda_{j}}(y-s)} \leq e^{T \sqrt{\lambda_{j}}},
$$

we get that

$$
\left\|\mathscr{I}_{2}(y)\right\|_{D\left(A^{\nu}\right)}^{2} \leq T \int_{0}^{T}\left(\sum_{j=1}^{\infty} \lambda_{j}^{2 \nu-1} e^{2 T \sqrt{\lambda_{j}}}\left|F_{j}(s)\right|^{2}\right) d s \leq T^{2}\|F\|_{L^{\infty}\left(0, T ; \mathscr{V}_{\nu-1 / 2, T}\right)}^{2}
$$

Therefore, we obtain that

$$
\left\|\mathscr{I}_{2}(y)\right\|_{D\left(A^{\nu}\right)} \leq T\|F\|_{L^{\infty}\left(0, T ; \mathscr{Y}_{\nu-1 / 2, T}\right)} .
$$

From the inequality (2.6), the third term $\mathscr{I}_{3}$ is estimated as follows

$$
\begin{aligned}
\left\|\mathscr{I}_{3}(y)\right\|_{D\left(A^{\nu}\right)}^{2} & =\sum_{j=1}^{\infty} \lambda_{j}^{2 \nu}\left(\frac{\cosh \left(\sqrt{\lambda_{j}} y\right)}{\cosh \left(\sqrt{\lambda_{j}} T\right)}\right)^{2}\left(\int_{0}^{T} \frac{\sinh \left(\sqrt{\lambda_{j}}(T-s)\right)}{\sqrt{\lambda_{j}}} F_{j}(s) d s\right)^{2} \\
& \leq 4 C_{\theta}(T-y)^{-2 \theta} \sum_{j=1}^{\infty} \lambda_{j}^{2 \nu} \lambda_{j}^{-\theta} T \int_{0}^{T}\left(\sinh \left(\sqrt{\lambda_{j}}(T-s)\right)\right)^{2}\left|F_{j}(s)\right|^{2} d s .
\end{aligned}
$$

Using (2.10), we find that

$$
\begin{aligned}
\left\|\mathscr{I}_{3}(y)\right\|_{D\left(A^{\nu}\right)}^{2} & \leq 4 T C_{\theta}(T-y)^{-2 \theta} \int_{0}^{T}\left(\sum_{j=1}^{\infty} \lambda_{j}^{2 \nu+\theta-1} e^{2 T \sqrt{\lambda_{j}}}\left|F_{j}(s)\right|^{2}\right) d s \\
& =4 T\left|C_{\theta}\right|^{2}(T-y)^{-2 \theta}\|F\|_{L^{\infty}\left(0, T ; \mathscr{Y}_{\nu-\theta / 2-1 / 2, T}\right)}^{2},
\end{aligned}
$$


which allows us to get that

$$
\left\|\mathscr{I}_{3}(y)\right\|_{D\left(A^{\nu}\right)} \leq 2 \sqrt{T} C_{\theta}(T-y)^{-\theta}\|F\|_{L^{\infty}\left(0, T ; \mathscr{H}_{\nu-\theta / 2-1 / 2, T}\right)} .
$$

Combining (2.5), 2.8), 2.12) and (2.15), we arrive at

$$
\begin{aligned}
\left\|u^{*}(y)\right\|_{D\left(A^{\nu}\right)} & \leq\left\|\mathscr{I}_{1}(y)\right\|_{D\left(A^{\nu}\right)}+\left\|\mathscr{I}_{2}(y)\right\|_{D\left(A^{\nu}\right)}+\left\|\mathscr{I}_{3}(y)\right\|_{D\left(A^{\nu}\right)} \\
& \leq 2 C_{\theta}(T-y)^{-\theta}\|f\|_{D\left(A^{\left.\nu-\frac{\theta}{2}\right)}\right.}+T\|F\|_{L^{\infty}\left(0, T ; \mathscr{V}_{\nu-1 / 2, T}\right)} \\
& +2 \sqrt{T} C_{\theta}(T-y)^{-\theta}\|F\|_{L^{\infty}\left(0, T ; \mathscr{V}_{\nu-\theta / 2-1 / 2, T}\right)} .
\end{aligned}
$$

This implies that

$$
\begin{aligned}
\int_{0}^{T}\left\|u^{*}(y)\right\|_{D\left(A^{\nu}\right)} d y & \leq 2 C_{\theta}\left(\int_{0}^{T}(T-y)^{-\theta} d y\right)\|f\|_{D\left(A^{\left.\nu-\frac{\theta}{2}\right)}\right.}+T^{2}\|F\|_{L^{\infty}\left(0, T ; \mathscr{Y}_{\nu-1 / 2, T}\right)} \\
& +2 \sqrt{T} C_{\theta}\left(\int_{0}^{T}(T-y)^{-\theta} d y\right)\|F\|_{L^{\infty}\left(0, T ; \mathscr{V}_{\nu-\theta / 2-1 / 2, T}\right)} .
\end{aligned}
$$

Hence, due to the proper integral $\int_{0}^{T}(T-y)^{-\theta} d y$ is convergent, we can deduce that $u^{*} \in L^{1}\left(0, T ; D\left(A^{\nu}\right)\right)$ and the following estimate holds

$$
\begin{aligned}
\left\|u^{*}\right\|_{L^{1}\left(0, T ; D\left(A^{\nu}\right)\right)} & \leq \frac{2 C_{\theta} T^{1-\theta}}{1-\theta}\|f\|_{D\left(A^{\left.\nu-\frac{\theta}{2}\right)}\right.}+T^{2}\|F\|_{L^{\infty}\left(0, T ; \mathscr{V}_{\nu-1 / 2, T}\right)} \\
& +\frac{2 \sqrt{T} C_{\theta} T^{1-\theta}}{1-\theta}\|F\|_{L^{\infty}\left(0, T ; \mathscr{Y}_{\nu-\theta / 2-1 / 2, T}\right)} .
\end{aligned}
$$

Theorem 2.2. Let $f$ and $F$ be as Theorem (2.1). Let $u^{\alpha, \epsilon}$ be the solution of ... Moreover, we have $\lim _{\epsilon \rightarrow 0} u^{1, \epsilon}=u^{*}$ and the following convergent is true

$$
\begin{aligned}
\left\|u^{1, \epsilon}-u^{*}\right\|_{L^{1}\left(0, T ; D\left(A^{\nu}\right)\right)} & \leq \sqrt{2 \epsilon} \frac{C_{\theta} e^{-\frac{\sqrt{\lambda_{1}} T}{2}} T^{1-\theta}}{1-\theta}\|f\|_{D\left(A^{\nu-\frac{\theta}{2}}\right)} \\
& +\sqrt{2 T \epsilon} \frac{C_{\theta} T^{1-\theta}}{1-\theta}\|F\|_{L^{\infty}\left(0, T ; \mathscr{W}_{\nu-\theta / 2-1 / 2, T}\right)} .
\end{aligned}
$$

Proof. We divide the proof into two parts.

Part 1. Existence and regularity of $u^{1, \epsilon}$.

Let us assume that $u(0)=u_{0} \in H$. Then we have the expression of $u$ as in Fourier series $u(y)=$ $\sum_{j=1}^{\infty}\left\langle u(y), \psi_{j}\right\rangle \psi_{j}$, where $\left\langle u(y), \psi_{j}\right\rangle$ is Fourier coefficient of $u$. Thanks to the work of [8], the Fourier coefficient of $u$ satisfies that the following equality

$$
\left\langle u(y), \psi_{j}\right\rangle=\cosh \left(\sqrt{\lambda_{j}} y\right)\left\langle u_{0}, \psi_{j}\right\rangle \cdot+\int_{0}^{y} \frac{\sinh \left(\sqrt{\lambda_{j}}(y-s)\right)}{\sqrt{\lambda_{j}}} F_{j}(s) d s .
$$

Hence

$$
\left\langle u(T), \psi_{j}\right\rangle=\cosh \left(\sqrt{\lambda_{j}} T\right)\left\langle u_{0}, \psi_{j}\right\rangle+\int_{0}^{T} \frac{\sinh \left(\sqrt{\lambda_{j}}(T-s)\right)}{\sqrt{\lambda_{j}}} F_{j}(s) d s .
$$

This implies that

$$
\begin{aligned}
\left\langle\alpha u(T)+\epsilon u(0), \psi_{j}\right\rangle & =\left(\alpha \cosh \left(\sqrt{\lambda_{j}} T\right)+\epsilon\right)\left\langle u_{0}, \psi_{j}\right\rangle \\
& +\alpha \int_{0}^{T} \frac{\sinh \left(\sqrt{\lambda_{j}}(y-s)\right)}{\sqrt{\lambda_{j}}} F_{j}(s) d s=\left\langle f, \psi_{j}\right\rangle .
\end{aligned}
$$


This implies that

$$
\begin{aligned}
\left\langle u_{0}, \psi_{j}\right\rangle & =\frac{\left\langle f, \psi_{j}\right\rangle}{\alpha \cosh \left(\sqrt{\lambda_{j}} T\right)+\epsilon} \\
& -\alpha\left(\cosh \left(\sqrt{\lambda_{j}} T\right)+\epsilon\right)^{-1} \int_{0}^{T} \frac{\sinh \left(\sqrt{\lambda_{j}}(T-s)\right)}{\sqrt{\lambda_{j}}} F_{j}(s) d s .
\end{aligned}
$$

By inserting the equation 2.23) into 2.20), we have immediately

$$
\begin{aligned}
\left\langle u^{\alpha, \epsilon}(y), \psi_{j}\right\rangle & =\frac{\cosh \left(\sqrt{\lambda_{j}} y\right)}{\alpha \cosh \left(\sqrt{\lambda_{j}} T\right)+\epsilon}\left\langle f, \psi_{j}\right\rangle+\int_{0}^{y} \frac{\sinh \left(\sqrt{\lambda_{j}}(y-s)\right)}{\sqrt{\lambda_{j}}} F_{j}(s) d s \\
& -\alpha \cosh \left(\sqrt{\lambda_{j}} y\right)\left(\cosh \left(\sqrt{\lambda_{j}} T\right)+\epsilon\right)^{-1} \int_{0}^{T} \frac{\sinh \left(\sqrt{\lambda_{j}}(T-s)\right)}{\sqrt{\lambda_{j}}} F_{j}(s) d s .
\end{aligned}
$$

By the properties of Fourier series, the mild solution to Problem 1.1 is given by

$$
\begin{aligned}
u^{\alpha, \epsilon}(y) & =\sum_{j=1}^{\infty} \frac{\cosh \left(\sqrt{\lambda_{j}} y\right)}{\alpha \cosh \left(\sqrt{\lambda_{j}} T\right)+\epsilon}\left\langle f, \psi_{j}\right\rangle \psi_{j}+\sum_{j=1}^{\infty}\left(\int_{0}^{y} \frac{\sinh \left(\sqrt{\lambda_{j}}(y-s)\right)}{\sqrt{\lambda_{j}}} F_{j}(s) d s\right) \psi_{j} \\
& -\alpha \sum_{j=1}^{\infty} \cosh \left(\sqrt{\lambda_{j}} y\right)\left(\alpha \cosh \left(\sqrt{\lambda_{j}} T\right)+\epsilon\right)^{-1}\left(\int_{0}^{T} \frac{\sinh \left(\sqrt{\lambda_{j}}(T-s)\right)}{\sqrt{\lambda_{j}}} F_{j}(s) d s\right) \psi_{j} \\
& =\mathscr{J}_{1}(y)+\mathscr{J}_{2}(y)+\mathscr{J}_{3}(y) .
\end{aligned}
$$

Using (2.6), we get the following estimate we get the following estimate

$$
\begin{aligned}
\left\|\mathscr{J}_{1}(y)\right\|_{D\left(A^{\nu}\right)}^{2} & \leq \frac{1}{\alpha^{2}} \sum_{j=1}^{\infty} \lambda_{j}^{2 \nu}\left(\frac{\cosh \left(\sqrt{\lambda_{j}} y\right)}{\cosh \left(\sqrt{\lambda_{j}} T\right)}\right)^{2}\left\langle f, \psi_{j}\right\rangle^{2} \\
& \leq \frac{4\left|C_{\theta}\right|^{2}}{\alpha^{2}}(T-y)^{-2 \theta} \sum_{j=1}^{\infty} \lambda_{j}^{2 \nu-\theta}\left\langle f, \psi_{j}\right\rangle^{2}=\frac{4\left|C_{\theta}\right|^{2}}{\alpha^{2}}(T-y)^{-2 \theta}\|f\|_{D\left(A^{\nu-\frac{\theta}{2}}\right)}^{2} .
\end{aligned}
$$

From the inequality (2.6), the third term $\mathscr{J}_{3}$ is estimated as follows

$$
\begin{aligned}
\left\|\mathscr{J}_{3}(y)\right\|_{D\left(A^{\nu}\right)}^{2} & =\sum_{j=1}^{\infty} \lambda_{j}^{2 \nu}\left(\frac{\alpha \cosh \left(\sqrt{\lambda_{j}} y\right)}{\alpha \cosh \left(\sqrt{\lambda_{j}} T\right)+\epsilon}\right)^{2}\left(\int_{0}^{T} \frac{\sinh \left(\sqrt{\lambda_{j}}(T-s)\right)}{\sqrt{\lambda_{j}}} F_{j}(s) d s\right)^{2} \\
& \leq 4 C_{\theta}(T-y)^{-2 \theta} \sum_{j=1}^{\infty} \lambda_{j}^{2 \nu} \lambda_{j}^{-\theta} T \int_{0}^{T}\left(\sinh \left(\sqrt{\lambda_{j}}(T-s)\right)\right)^{2}\left|F_{j}(s)\right|^{2} d s \\
& \leq 4 T\left|C_{\theta}\right|^{2}(T-y)^{-2 \theta}\|F\|_{L^{\infty}\left(0, T ; \mathscr{Y}_{\nu-\theta / 2-1 / 2, T}\right)}^{2}
\end{aligned}
$$

Therefore, we can deduce that

$$
\left\|\mathscr{J}_{3}(y)\right\|_{D\left(A^{\nu}\right)} \leq 2 \sqrt{T} C_{\theta}(T-y)^{-\theta}\|F\|_{L^{\infty}\left(0, T ; \mathscr{V}_{\nu-\theta / 2-1 / 2, T}\right)} .
$$

Part 2. The convergence of $u^{1, \epsilon}$ and $u *$ when $\epsilon \rightarrow 0$.

When $\alpha=1$, we have the following fomula

$$
\begin{aligned}
u^{1, \epsilon}(y) & =\sum_{j=1}^{\infty} \frac{\cosh \left(\sqrt{\lambda_{j}} y\right)}{\cosh \left(\sqrt{\lambda_{j}} T\right)+\epsilon}\left\langle f, \psi_{j}\right\rangle \psi_{j}+\sum_{j=1}^{\infty}\left(\int_{0}^{y} \frac{\sinh \left(\sqrt{\lambda_{j}}(y-s)\right)}{\sqrt{\lambda_{j}}} F_{j}(s) d s\right) \psi_{j} \\
& -\sum_{j=1}^{\infty} \cosh \left(\sqrt{\lambda_{j}} y\right)\left(\cosh \left(\sqrt{\lambda_{j}} T\right)+\epsilon\right)^{-1}\left(\int_{0}^{T} \frac{\sinh \left(\sqrt{\lambda_{j}}(T-s)\right)}{\sqrt{\lambda_{j}}} F_{j}(s) d s\right) \psi_{j} .
\end{aligned}
$$


Since the representations of $u^{1, \epsilon}$ and $u^{*}$, we find that

$$
\begin{aligned}
& u^{1, \epsilon}(y)-u^{*}(y) \\
& =\sum_{j=1}^{\infty}\left(\frac{\cosh \left(\sqrt{\lambda_{j}} y\right)}{\cosh \left(\sqrt{\lambda_{j}} T\right)}-\frac{\cosh \left(\sqrt{\lambda_{j}} y\right)}{\cosh \left(\sqrt{\lambda_{j}} T\right)+\epsilon}\right)\left\langle f, \psi_{j}\right\rangle \psi_{j} \\
& -\sum_{j=1}^{\infty}\left(\frac{\cosh \left(\sqrt{\lambda_{j}} y\right)}{\cosh \left(\sqrt{\lambda_{j}} T\right)}-\frac{\cosh \left(\sqrt{\lambda_{j}} y\right)}{\cosh \left(\sqrt{\lambda_{j}} T\right)+\epsilon}\right)\left(\int_{0}^{T} \frac{\sinh \left(\sqrt{\lambda_{j}}(T-s)\right)}{\sqrt{\lambda_{j}}} F_{j}(s) d s\right) \psi_{j} .
\end{aligned}
$$

By a simple caculation, we obtain

$$
\begin{aligned}
& u^{1, \epsilon}(y)-u^{*}(y) \\
& =\sum_{j=1}^{\infty} \frac{\epsilon \cosh \left(\sqrt{\lambda_{j}} y\right)}{\cosh \left(\sqrt{\lambda_{j}} T\right)\left(\cosh \left(\sqrt{\lambda_{j}} T\right)+\epsilon\right)}\left\langle f, \psi_{j}\right\rangle \psi_{j} \\
& -\sum_{j=1}^{\infty} \frac{\epsilon \cosh \left(\sqrt{\lambda_{j}} y\right)}{\cosh \left(\sqrt{\lambda_{j}} T\right)\left(\cosh \left(\sqrt{\lambda_{j}} T\right)+\epsilon\right)}\left(\int_{0}^{T} \frac{\sinh \left(\sqrt{\lambda_{j}}(T-s)\right)}{\sqrt{\lambda_{j}}} F_{j}(s) d s\right) \psi_{j} \\
& =\mathscr{K}_{1}(y)+\mathscr{K}_{2}(y) .
\end{aligned}
$$

Now, we focus on the first term $\mathscr{K}_{1}$. Using the inequality

$$
\cosh \left(\sqrt{\lambda_{j}} T\right)+\epsilon \geq 2 \sqrt{\epsilon} \sqrt{\cosh \left(\sqrt{\lambda_{j}} T\right)}
$$

we find that

$$
\begin{aligned}
\frac{\epsilon \cosh \left(\sqrt{\lambda_{j}} y\right)}{\cosh \left(\sqrt{\lambda_{j}} T\right)\left(\cosh \left(\sqrt{\lambda_{j}} T\right)+\epsilon\right)} & \leq \frac{\sqrt{\epsilon}}{2} \frac{\cosh \left(\sqrt{\lambda_{j}} y\right)}{\cosh ^{3 / 2}\left(\sqrt{\lambda_{j}} T\right)} \leq \sqrt{2 \epsilon} \frac{e^{\sqrt{\lambda_{j}} y}}{e^{\frac{3 \sqrt{\lambda_{j}} T}{2}}} \\
& \leq \sqrt{2 \epsilon} e^{-\frac{\sqrt{\lambda_{1}} T}{2}} e^{\sqrt{\lambda_{j}}(y-T)}
\end{aligned}
$$

By looking at the inequality $e^{-z} \leq C_{\theta} z^{-\theta}$, we obtain the following estimate

$$
\frac{\epsilon \cosh \left(\sqrt{\lambda_{j}} y\right)}{\cosh \left(\sqrt{\lambda_{j}} T\right)\left(\cosh \left(\sqrt{\lambda_{j}} T\right)+\epsilon\right)} \leq C_{\theta} \sqrt{2 \epsilon} e^{-\frac{\sqrt{\lambda_{1}} T}{2}} \lambda_{j}^{-\theta / 2}(T-y)^{-\theta} .
$$

This implies that

$$
\begin{aligned}
\left\|\mathscr{K}_{1}(y)\right\|_{D\left(A^{\nu}\right)}^{2} & =\sum_{j=1}^{\infty} \lambda_{j}^{2 \nu}\left(\frac{\epsilon \cosh \left(\sqrt{\lambda_{j}} y\right)}{\cosh \left(\sqrt{\lambda_{j}} T\right)\left(\cosh \left(\sqrt{\lambda_{j}} T\right)+\epsilon\right)}\right)^{2}\left\langle f, \psi_{j}\right\rangle^{2} \\
& \leq 2 \epsilon\left|C_{\theta}\right|^{2} e^{-\sqrt{\lambda_{1}} T}(T-y)^{-2 \theta} \sum_{j=1}^{\infty} \lambda_{j}^{2 \nu-\theta}\left\langle f, \psi_{j}\right\rangle^{2} \\
& =2 \epsilon\left|C_{\theta}\right|^{2} e^{-\sqrt{\lambda_{1}} T}(T-y)^{-2 \theta}\|f\|_{D\left(A^{\nu-\frac{\theta}{2}}\right)}^{2}
\end{aligned}
$$

Hence, we derive the following estimate

$$
\left\|\mathscr{K}_{1}(y)\right\|_{D\left(A^{\nu}\right)} \leq \sqrt{2 \epsilon} C_{\theta} e^{-\frac{\sqrt{\lambda_{1}} T}{2}}(T-y)^{-\theta}\|f\|_{D\left(A^{\nu-\frac{\theta}{2}}\right)} .
$$


Next, we continue to treat the second term $\mathscr{K}_{2}(y)$. Using $(2.33)$ and Hölder inequality, it it easy to observe that

$$
\begin{aligned}
\left\|\mathscr{K}_{2}(y)\right\|_{D\left(A^{\nu}\right)}^{2} & =\sum_{j=1}^{\infty} \lambda_{j}^{2 \nu}\left(\frac{\epsilon \cosh \left(\sqrt{\lambda_{j}} y\right)}{\cosh \left(\sqrt{\lambda_{j}} T\right)\left(\cosh \left(\sqrt{\lambda_{j}} T\right)+\epsilon\right)}\right)^{2}\left(\int_{0}^{T} \frac{\sinh \left(\sqrt{\lambda_{j}}(T-s)\right)}{\sqrt{\lambda_{j}}} F_{j}(s) d s\right)^{2} \\
& \leq 2 \epsilon\left|C_{\theta}\right|^{2} e^{-\sqrt{\lambda_{1}} T}(T-y)^{-2 \theta} \sum_{j=1}^{\infty} \lambda_{j}^{2 \nu-\theta-1} T \int_{0}^{T}\left(\sinh \left(\sqrt{\lambda_{j}}(T-s)\right)\right)^{2}\left|F_{j}(s)\right|^{2} d s \\
& \leq 2 \epsilon\left|C_{\theta}\right|^{2} e^{-\sqrt{\lambda_{1}} T} T(T-y)^{-2 \theta}\|F\|_{L^{\infty}\left(0, T ; \mathscr{Y}_{\nu-\theta / 2-1 / 2, T}\right.}^{2} .
\end{aligned}
$$

Hence, we get that

$$
\left\|\mathscr{K}_{2}(y)\right\|_{D\left(A^{\nu}\right)} \leq \sqrt{2 T \epsilon} C_{\theta}(T-y)^{-\theta}\|F\|_{L^{\infty}\left(0, T ; \mathscr{V}_{\nu-\theta / 2-1 / 2, T}\right)} .
$$

Combining (2.31), 2.35) and 2.37), we find that

$$
\begin{aligned}
\left\|u^{1, \epsilon}(y)-u^{*}(y)\right\|_{D\left(A^{\nu}\right)} & \leq\left\|\mathscr{K}_{1}(y)\right\|_{D\left(A^{\nu}\right)}+\left\|\mathscr{K}_{2}(y)\right\|_{D\left(A^{\nu}\right)} \\
& \leq \sqrt{2 \epsilon} C_{\theta} e^{-\frac{\sqrt{\lambda_{1}} T}{2}}(T-y)^{-\theta}\|f\|_{D\left(A^{\nu-\frac{\theta}{2}}\right)} \\
& +\sqrt{2 T \epsilon} C_{\theta}(T-y)^{-\theta}\|F\|_{L^{\infty}\left(0, T ; \mathscr{V}_{\nu-\theta / 2-1 / 2, T}\right)} .
\end{aligned}
$$

This implies that the following estimate

$$
\begin{aligned}
\int_{0}^{T}\left\|u^{1, \epsilon}(y)-u^{*}(y)\right\|_{D\left(A^{\nu}\right)} d y & \leq \sqrt{2 \epsilon} C_{\theta} e^{-\frac{\sqrt{\lambda_{1}} T}{2}}\left(\int_{0}^{T}(T-y)^{-\theta} d y\right)\|f\|_{D\left(A^{\nu-\frac{\theta}{2}}\right)} \\
& +\sqrt{2 T \epsilon} C_{\theta}\left(\int_{0}^{T}(T-y)^{-\theta} d y\right)\|F\|_{L^{\infty}\left(0, T ; \mathscr{V}_{\nu-\theta / 2-1 / 2, T}\right)} .
\end{aligned}
$$

Since the proper integral $\int_{0}^{T}(T-y)^{-\theta} d y$ is convergent $(0<\theta<1)$, we know that

$$
\begin{aligned}
\left\|u^{1, \epsilon}-u^{*}\right\|_{L^{1}\left(0, T ; D\left(A^{\nu}\right)\right)} & \leq \sqrt{2 \epsilon} \frac{C_{\theta} e^{-\frac{\sqrt{\lambda_{1}} T}{2}} T^{1-\theta}}{1-\theta}\|f\|_{D\left(A^{\nu-\frac{\theta}{2}}\right)} \\
& +\sqrt{2 T \epsilon} \frac{C_{\theta} T^{1-\theta}}{1-\theta}\|F\|_{L^{\infty}\left(0, T ; \mathscr{W}_{\nu-\theta / 2-1 / 2, T}\right)} .
\end{aligned}
$$

\section{References}

[1] H.N. Dinh, D.V. Nguyen, H. Sahli, A non-local boundary value problem method for the Cauchy problem for elliptic equations, Inverse Problems, 25:055002, 2009.

[2] M.M. Lavrentev, V.G. Romanov, and S.P. Shishatskii, Ill-posed problems of mathematical physics and analysis, Translations of Mathematical Monographs, Vol. 64, American Mathematical Society, Providence, RI, 1986.

[3] C.R. Johnson, Computational and numerical methods for bioelectric field problems, Crit. Rev. Biomed. Eng. 25 (1997), pp. $1-81$.

[4] R. Gorenflo, Funktionentheoretische bestimmung des aussenfeldes zu einer zweidimensionalen magnetohydrostatischen konfiguration, Z. Angew. Math. Phys. 16 (1965), pp. 279-290.

[5] J. Hadamard, Lectures on the Cauchy Problem in Linear Differential Equations, Yale University Press, New Haven, CT, 1923.

[6] T. Hohage. Regularization of exponentially ill-posed problems, Numer. Funct. Anal. Optim., 21(3-4):439-464, 2000.

[7] V. Isakov, Inverse Problems for Partial Differential Equations, Volume 127 of Applied Mathematical Sciences, Springer, New York, second edition, 2006.

[8] N.H. Tuan, D.D. Trong, P.H. Quan, A note on a Cauchy problem for the Laplace equation: regularization and error estimates, Appl. Math. Comput. 217 (2010), no. 7, 2913-2922. 
[9] N.A. Tuan, D. O'regan, D. Baleanu, N.H. Tuan, On time fractional pseudo-parabolic equations with nonlocal integral conditions, Evolution Equation and Control Theory, 10.3934/eect.2020109.

[10] N.H. Can, N.H. Tuan, V.V. Au, L.D. Thang, Regularization of Cauchy abstract problem for a coupled system for nonlinear elliptic equations, J. Math. Anal. Appl. 462 (2018), no. 2, 1148-1177.

[11] M.M. Lavrentev, V.G. Romanov, and S.P. Shishatskii, Ill-posed problems of mathematical physics and analysis, Translations of Mathematical Monographs, Vol. 64, American Mathematical Society, Providence, RI, 1986.

[12] C.R. Johnson, Computational and numerical methods for bioelectric field problems, Crit. Rev. Biomed. Eng. 25 (1997), pp. $1-81$.

[13] V.A. Khoa, M.T.N. Truong, N.H.M. Duy, N.H. Tuan, The Cauchy problem of coupled elliptic sine-Gordon equations with noise: Analysis of a general kernel-based regularization and reliable tools of computing, Comput. Math. Appl. 73 (2017), no. $1,141-162$.

[14] N.H. Tuan, L.D. Thang, D. Lesnic, A new general filter regularization method for Cauchy problems for elliptic equations with a locally Lipschitz nonlinear source, J. Math. Anal. Appl. 434 (2016), no. 2, 1376-1393.

[15] B. Kaltenbacher, A. Kirchner, B. Vexler, Adaptive discretizations for the choice of a Tikhonov regularization parameter in nonlinear inverse problems, Inverse Problems 27 (2011) 125008.

[16] B. Kaltenbacher, W. Polifke, Some regularization methods for a thermoacoustic inverse problem, in: Special Issue M.V. Klibanov, J. Inverse Ill-Posed Probl. 18 (2011) 997-1011.

[17] B. Kaltenbacher, F. Schoepfer, Th. Schuster, Convergence of some iterative methods for the regularization of nonlinear ill-posed problems in Banach spaces, Inverse Problems 25 (2009) 065003, 19 pp.

[18] M. Asaduzzaman, Existence Results for a Nonlinear Fourth Order Ordinary Differential Equation with Four-Point Boundary Value Conditions. Advances in the Theory of Nonlinear Analysis and its Application , 4 (4) (2020), 233-242

[19] M. Massar, On a fourth-order elliptic Kirchhoff type problem with critical Sobolev exponent, Advances in the Theory of Nonlinear Analysis and its Application , 4 (4), 394-401

[20] I. Kim, Semilinear problems involving nonlinear operators of monotone type, Results in Nonlinear Analysis , 2 (1)(2009), $25-35$

[21] S. Weng, X. Liu, Z. Chao, Some Fixed Point Theorems for Cyclic Mapping in a Complete $b$-metric-like Space, Results in Nonlinear Analysis , 2020 V3 I4 , 207-213 